\title{
Processo Participativo de Engenharia de Requisitos para Construção de Tecnologias Educacionais: Versão Preliminar
}

\author{
Ramon Pontes Araújo', Francisco Petrônio A. Medeiros ${ }^{2}$ \\ Programa de Pós-Graduação em Tecnologia da Informação - Instituto Federal da \\ Paraíba (IFPB) João Pessoa - PB - Brasil \\ ramon . pontes @academico . ifpb.edu . br ${ }^{1}$; petronio@ ifpb.edu . br ${ }^{2}$
}

\begin{abstract}
There are many methods and techniques for eliciting and specifying general-purpose software requirements in the literature and professional practice. A feature perceived from a systematic mapping of the literature was the need to consider the use of pedagogical practices developed by teachers and students and the active participation of these stakeholders in the design of the educational technology solution. This work aims to propose a participatory requirements engineering process for constructing educational technologies from the theoretical frameworks of Problem-Based Learning, Design Thinking, and Participatory Design.
\end{abstract}

Resumo. Na literatura e prática profissional há uma grande quantidade de métodos e técnicas para elicitação e especificação de requisitos de software de propósito geral. Uma característica percebida a partir de um mapeamento sistemático da literatura foi a necessidade de considerar o uso das práticas pedagógicas desenvolvidas por professores e alunos, bem como a participação ativa desses stakeholders no projeto da solução das tecnologias educacionais. Como forma de investigar essas lacunas, este trabalho tem como objetivo propor um processo participativo de engenharia de requisitos para a construção de tecnologias educacionais a partir dos referenciais teóricos de Aprendizagem Baseada em Problemas, Design Thinking e Design Participativo.

\section{Introdução}

A inserção de tecnologias da informação e comunicação (TIC) no contexto educacional é uma realidade, onde softwares e aplicativos com objetivos gerais ou específicos contribuem para a aprendizagem. Zhao et al. (2021) destacam que essas tecnologias são percebidas como ferramentas que facilitam o aprendizado e desenvolvem as habilidades do aluno, oferecendo a eles modos alternativos ao aprendizado tradicional, ao mesmo tempo que impõem novas competências aos professores. No campo educacional, o uso destas tecnologias só tem propósito à medida que os professores a percebem como uma aliada, ou seja, como uma ferramenta que auxilie suas atividades didáticos-pedagógicas e, que, os alunos a vejam como um elemento que os motiva e ao mesmo tempo os desafie com o surgimento de novos caminhos. Com destaque para as áreas da engenharia de requisitos que permitem tornar os processos de ensino e de aprendizagem inovadores, participativos e interativos [Teixeira et al. 2003].

A engenharia de requisitos (ER) contribui na concepção de tecnologias para os mais variados propósitos, sendo fundamental para que funcionem de acordo com as necessidades dos usuários e restrições sob as quais se encontre submetido. Quando a tecnologia se destina ao contexto educacional, que envolve aspectos cognitivos e sociais específicos, novas variáveis são incorporadas ao desenvolvimento de modo que a tecnologia criada engaje os estudantes e professores. A tecnologia também precisa estar incorporada metodologicamente ao processo de ensino aprendizagem.

Diante da especificidade do uso dessas tecnologias, Perry (2005) discute que a dimensão educacional aplicada à produção de tecnologias (incorporação de novos 
requisitos, manutenção, usabilidade, design de interface) torna maior o desafio para os desenvolvedores porque não é possível simplesmente transpor uma dada teoria que oriente a prática pedagógica para a tecnologia a ser criada. Diante da especificidade do uso destas tecnologias, é sugerido atentar a características não limitantes ao aprendizado do aluno e às práticas pedagógicas dos professores, contribuindo para formação e evolução do conhecimento, incentivando a análise, argumentação e pensamento crítico.

Para Souto e Silva (2020), a falta de alinhamento das práticas pedagógicas com as tecnologias educacionais contribui negativamente para o engajamento e uso mais efetivo que essas ferramentas poderiam proporcionar. Para Neto et al. (2003), a chave para o desenvolvimento de sistemas colaborativos voltados ao contexto educacional está no uso de um processo adequado de engenharia de requisitos, pois é nesta fase da engenharia de software onde as necessidades dos usuários são identificadas e as funcionalidades do sistema são modeladas. Uma das recomendações para se alcançar requisitos mais adequados, segundo Silva et al. (2012), é envolver os alunos no processo de desenvolvimento através de abordagens de projetos participativos.

\section{Trabalhos Relacionados}

Foi realizado um mapeamento sistemático da literatura com o objetivo de levantar o estado da arte relacionando processos de engenharia de requisitos para construção de tecnologias educacionais (Araújo e Medeiros, 2020). Dos 173 trabalhos retornados no mapeamento, 32 foram analisados em detalhes, aos quais se destacam os quatro mais relacionados com a proposta desta pesquisa, uma vez que abordam aspectos cognitivos, sociais e de elementos humanos que vão além das práticas tradicionais. Carrión et al. (2017) analisam que usualmente as tecnologias educacionais desenvolvidas são resultado da aplicação de uma metodologia de design que não aborda aspectos pedagógicos, e esses aspectos só são percebidos quando se permite a participação ativa já na fase de requisitos, utilizando técnicas que proporcionam a construção de um produto a partir de um conhecimento multidisciplinar que se sobrepõe ao conhecimento individual. Wang (2005) apresenta um modelo interativo entre alunos e professores, em ciclos de decisão de âmbito coletivo, que buscam aprimorar um plano inicial de projeto até a implementação final, no entanto esse modelo não contempla as práticas pedagógicas nem detalha de forma estrutural as fases destes ciclos e suas atividades.

Santa-Rosa e Struchiner (2010) destacam que a participação do usuário no design não deve se restringir apenas às fases de testes de protótipos ou avaliação. Os autores sugerem um processo com três fases para a realização e desenvolvimento de ambientes virtuais de aprendizagem. $O$ trabalho foca no processo específico para $\mathrm{o}$ desenvolvimento de um AVA na área de Histologia e sua avaliação de usabilidade, não apresentando as etapas que compõem o processo em detalhes para uma possível replicação. Perry (2005) propõe uma metodologia participativa para desenvolvimento de softwares educacionais. A metodologia envolve as fases de projeto educacional, implementação e avaliação. As etapas relacionadas ao projeto educacional não são detalhadas e os stakeholders envolvidos na metodologia não incluem os estudantes, somente os professores e demais envolvidos da área de Engenharia de Software.

Com base no mapeamento sistemático da literatura realizado, bem como pesquisas ad-hoc conduzidas, não foram encontrados processos de engenharia de requisitos para construção de tecnologias educacionais que seguissem os princípios do design participativo de forma sistêmica, nem considerassem aspectos pedagógicos nas etapas de elicitação e especificação dos requisitos. Considerando essa análise, esse trabalho objetiva estabelecer um processo participativo de engenharia de requisitos para a construção de tecnologias educacionais que leve em consideração as lacunas apontadas na literatura. 


\section{Método}

O mapeamento sistemático conduzido por Araújo e Medeiros (2020) levantou um conjunto de técnicas e métodos de Engenharia de Requisitos pesquisadas e aplicadas no contexto do desenvolvimento de tecnologias educacionais, as doze mais citadas foram: análise documental, pesquisa bibliográfica, etnografia, teoria da atividade, grupo focal, entrevistas, prototipação, brainstorming, questionários, observação, modelo conceitual e cenários. Como percebido, tais técnicas não diferem exatamente de outros contextos, que não o educacional, em relação aos processos de engenharia de requisitos utilizados. Esses trabalhos analisados, em sua grande maioria, utilizaram tais técnicas para o desenvolvimento de tecnologias como objetos de aprendizagem, AVAs, jogos, modelos de aprendizagem e até mesmo protótipos de alta fidelidade.

Ao mesmo tempo, a partir dos trabalhos analisados, foi possível chegar a três conclusões que fundamentam a proposição do processo participativo de engenharia de requisitos para construção de tecnologias educacionais apresentado neste trabalho, a saber: (i) considerar os aspectos pedagógicos no processo de design de modo a alcançar a construção de tecnologias que suportem uma aprendizagem mais significativa; (ii) envolver estudantes e professores no processo de desenvolvimento através de abordagens de projetos participativos; e (iii) compreender que as técnicas de engenharia de requisitos utilizadas no desenvolvimento das tecnologias educacionais ou a própria combinação delas não são o fator mais importante quanto aos objetivos de aprendizagem e sucesso da tecnologia desenvolvida, mas sim a orquestração e alcance dos envolvidos no processo, a caracterização do problema e a identificação dos perfis de usuários e de aprendizagem abordados pelas tecnologias desenvolvidas.

Com base nas conclusões do mapeamento sistemático, um grupo de pesquisadores das áreas de Engenharia de Software e Metodologias de Aprendizagem Ativas têm desenvolvido, iterativamente, um processo de engenharia de requisitos para a construção de tecnologias educacionais. O percurso metodológico definido para alcançar este objetivo, além do mapeamento sistemático previamente citado, contém: (1) a escolha dos referenciais teórico-metodológicos que dariam suporte ao processo, (2) a elaboração iterativa e incremental de uma versão preliminar do processo e por fim, (3) o refinamento e validação do processo por meio de workshops e questionários em ambientes educacionais reais. Esse artigo tem como objetivo descrever as etapas 1 e 2 .

\section{Resultados}

Os referenciais teórico-metodológicos para dar suporte à elaboração do processo deveriam considerar as conclusões levantadas no mapeamento sistemático. Para a inclusão de aspectos pedagógicos no processo foi escolhida a metodologia de Aprendizagem baseada em Problemas, que possui como características a postura ativa dos estudantes, a iteratividade e a resolução de problemas facilitada pelo professor (Oliveira et al. 2020). Para envolver os stakeholders do contexto educacional optou-se por espelhar o processo no design participativo, que preconiza a integração dos professores e estudantes aos profissionais de engenharia de software no efetivo desenvolvimento da tecnologia (DiSalvo et al. 2017). Objetivando orquestrar os protagonistas com perfis heterogêneos no processo de desenvolvimento, decidiu-se por referenciar o framework Design Thinking, que é uma abordagem já disseminada em diferentes áreas de conhecimento e alinhada aos métodos de desenvolvimento ágil.

Levantou-se a hipótese de que o uso de um processo ativo participativo aplicado a um problema definido e organizado sistematicamente poderia contribuir efetivamente para a criação de tecnologias educacionais mais efetivas, que por consequência estimulariam o engajamento dos alunos e professores. O processo participativo proposto envolve práticas pedagógicas já aplicadas em sala de aula, através de um processo que define atividades para os diversos envolvidos em busca de uma solução do problema, como apresentado na Figura 1. O processo tem início com a fase 1 (Projeto 
Educacional), fase preliminar que antecede a fase workshop participativo. Embasada nos modelos de Aprendizagem baseada em Problemas apresentados por Ribeiro (2008) e Hung (2009), que permite ao aluno a experiência da resolução de problemas, nesta fase os especialistas educacionais abordam a situação real do problema imergindo os envolvidos dentro do contexto que será abordado. Essa etapa deve ser apoiada pelo Engenheiro de Software, responsável por dar suporte aos especialistas em educação envolvidos no desenvolvimento da tecnologia educacional, organizando e sintetizando o problema, ideias e possível proposição do tipo de tecnologia que deverá ser produzida.

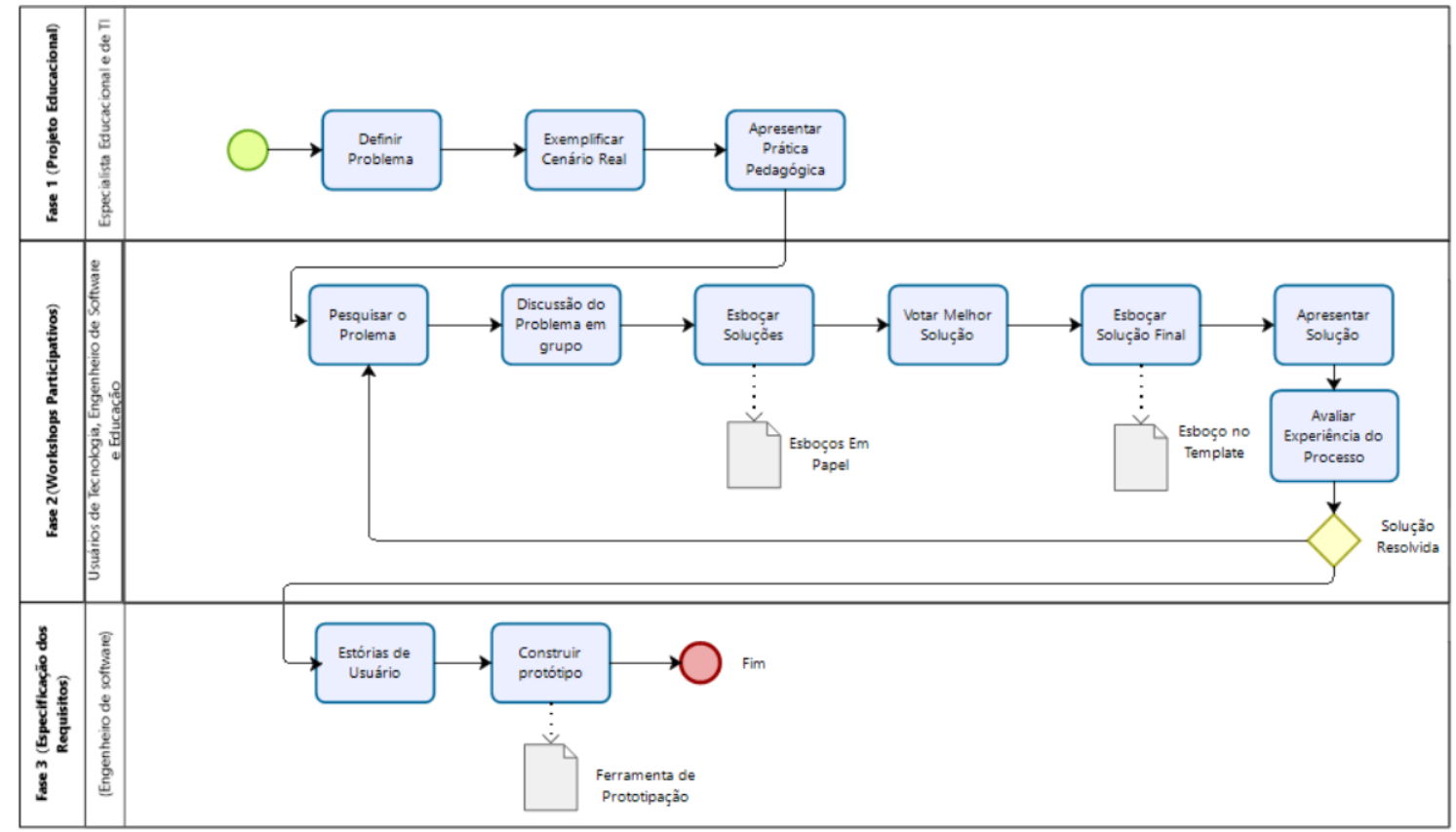

Figura 1. Processo Participativo para Construção de Softwares Educacionais

A primeira atividade consiste na definição do problema. Considera-se que durante esta atividade o especialista pode exemplificar cenários reais do problema, a ideia é tornar mais claro o entendimento para todos os envolvidos. Ainda nessa fase os especialistas educacionais devem apresentar práticas pedagógicas que já utilizam em sala de aula que podem, se utilizadas, ajudar na resolução do problema em questão. Uma vez que todos os especialistas educacionais envolvidos, sejam professores, tutores, coordenadores, diretores, pesquisadores, etc. compreendem a existência do problema e entendem a importância de solucioná-lo, ao mesmo tempo que conseguem descrever o problema para o Engenheiro de Software responsável por conduzir e orientar as duas próximas fases do processo participativo, este segue para a fase 2 (Workshops Participativos). Estima-se que a Fase 1 tenha duração de 30 minutos por seção. Mais de uma seção pode ser necessária por decisão dos stakeholders envolvidos.

Ferreira, Couto e Oswald (2020) definem que os workshops possibilitam construções coletivas e colaborativas de conhecimento, já que todos participam e contribuem com seu trabalho, suas ações, reflexões, argumentações, análises. Definiu-se o workshop como arcabouço metodológico para a fase 2 do processo proposto, tendo como expectativa a transformação do pesquisador e dos sujeitos envolvidos a partir da abordagem seguida para sua execução, pois, se é uma atividade que provoca mudança nos envolvidos em seu processo, é porque está pautada em uma abordagem históricocultural. A fase 2 indica que sejam criados grupos de no máximo cinco estudantes com o professor intercambiando entre os grupos, apoiando as suas escolhas, sanando as dúvidas e intervindo quando necessário. Os alunos em grupos separados iniciam com a atividade de pesquisar o problema com tempo de duração de vinte minutos, visando conhecer mais do(s) tópico(s) abordado(s) na tecnologia, gerar dúvidas e aguçar a 
criatividade. Para a próxima atividade, quinze minutos destinados a discussão do problema em grupo com a finalidade de organizarem as ideias com base no resultado de suas pesquisas. Espera-se que o(s) tópico(s) a ser(em) abordados na tecnologia desenvolvida esteja sendo ou já tenha sido estudada pelos estudantes.

Seguindo o processo, os alunos são orientados a esboçar soluções de forma individual utilizando um esboço em papel que será distribuído entre os grupos, com duração de vinte minutos, seguindo para a atividade votar melhor solução, onde será escolhido entre os participantes de cada grupo a(s) melhor(es) solução(es), no máximo duas, com duração de 5 minutos. Para a atividade de votar a melhor solução cada aluno terá dois votos que poderão ser distribuídos entre as soluções criadas dentro do seu próprio grupo. Um especialista Engenharia de Software, que estará observando e monitorando as rodadas de execução das atividades, fará a contagem da solução mais votada. Após a votação da(s) melhor(es) solução(ões), cada grupo terá a oportunidade de esboçar solução final com a participação de todos do grupo para refinar a solução escolhida, com duração de 25 minutos, em templates que serão fornecidos pelo Engenheiro de Software. Na sequência, os alunos escolherão um representante de cada grupo para apresentar solução, com duração de 5 minutos por apresentação. Caso o grupo, após a votação, decida por esboçar a solução final de duas soluções, o grupo pode se dividir para desenvolvê-las. Ao final, somente uma das soluções é apresentada.

Ao final do workshop, os participantes participarão de um grupo focal para avaliar a experiência do processo, apontar limitações e sugerir melhorias. A análise dos grupos focais servirá para ajustes no processo para os workshops vindouros, bem como para validação empírica do processo na terceira etapa da metodologia. O grupo focal terá uma duração de quinze minutos. Além de questões analisando as limitações e melhorias do processo, também discutirá e analisará o sentimento de pertencimento e engajamento dos estudantes, assim como o processo de colaboração e ideação da solução para o problema vivenciado pelos envolvidos durante o processo. Ao final dessa pesquisa, quando o processo participativo de engenharia de requisitos para construção de tecnologias educacionais estiver validado, essa etapa poderá ser reduzida ou mesmo suprimida pelo engenheiro de software, visto que o processo não estará mais em fase de desenvolvimento, ao mesmo tempo que o grupo focal possa servir para coletar mais alguns dados dos participantes de modo a apoiar a fase 3 do processo.

As soluções finais apresentadas pelos alunos são avaliadas pelo professor, com o apoio do engenheiro de software, que indicarão se o problema foi solucionado, ou seja, se os esboços das tecnologias educacionais desenvolvidas atendem às expectativas. Se sim, ela seguirá para a fase 3 (Especificação dos Requisitos). A fase 3 (Especificação dos Requisitos) apresenta atividades que serão desenvolvidas em um momento posterior ao Workshop pelo Engenheiro de Software, sintetizando tudo que foi elaborado pelos participantes na fase 2 . As estórias de usuários serão desenvolvidas com base no que foi apresentado e discutido pelos alunos, ensejando organizar todos os detalhes e funcionalidades apresentados no software. Os materiais produzidos em todas as etapas do workshop pelos alunos e especialistas educacionais serão considerados como documentação a fase 3 . Por fim, a atividade construir protótipos de média ou alta fidelidade navegável, utilizando softwares especializados, materializa o projeto final.

\section{Trabalhos Futuros e Conclusão}

Considerando que a pesquisa está em andamento, já foram concluídas as três primeiras etapas metodológicas, que foram o mapeamento sistemático, levantamento dos referenciais teórico-metodológicos para elaboração do processo, elaboração iterativa e incremental de uma versão preliminar do processo. A pesquisa iniciou a condução dos workshops de refinamento do processo de ER proposto, e finalmente será concluída com a validação dos protótipos desenvolvimentos em cada umas das instanciações do processo por meio de um questionário de aceitação da tecnologia educacional proposta com base no modelo TAM - Technology Acceptance Model (Kind and He, 2006). 


\section{Agradecimentos}

Gostaríamos de agradecer o apoio financeiro do Instituto Federal da Paraíba Campus João Pessoa para condução dessa pesquisa.

\section{Referências}

Araújo, R. P. and Medeiros, F. P. A. "Requirements Elicitation and Specification for Educational Technology Development: A Systematic Literature Mapping," (2020). 15th Iberian Conference on Information Systems and Technologies (CISTI), Sevilla, Spain, 2020, pp. 1-6, doi: 10.23919/CISTI49556.2020.9141015.

Carrión, M., Santórum, M., Pérez, M. and Aguilar, J. (2017). "A participatory methodology for the design of serious games in the educational environment". Congreso Internacional de Innovación y Tendencias en Ingeniería (CONIITI), Bogotá, 2017, pp. 1-6, doi: 10.1109/CONIITI.2017.8273363.

DiSalvo, B., Yip, J., Bonsignore, E. and DiSalvo, C. (2017) "Participatory design for learning”. Routledge.

Ferreira, H. M. C., Couto Jr, D. R., \& Oswald, M. L. M. B. (2020). “As oficinas como lócus de encontro com o outro: uma abordagem histórico-cultural. Metodologia de pesquisa científica em Informática na Educação: abordagem qualitativa, SBC”. Disponível em:< https://metodologia. ceie-br. org/livro-3.

Neto, G. G., Gomes, A. S., Tedesco, P. (2003). "Elicitação de Requisitos de Sistemas Colaborativos de Aprendizagem Centrada na Atividade de Grupo". Simpósio Brasileiro de Informática na Educação - SBIE, p. 317-326.

Hung, W. (2009). "The 9-step problem design process for problem-based learning: Application of the 3C3R model". University of North Dakota, Instructional Design \& Technology, Education Building Room 218, 231 Centennial Drive STOP 7189, Grand Forks, ND 58202-7189, United States.

King, W. R., and He, J. (2006) "A meta-analysis of the technology acceptance model." Information \& management 43, no. 6, 740-755.

Oliveira, M. A., Medeiros, F. P. and Melo, L. B. (2020) "Levantamento do Estado da Arte sobre Aprendizagem baseada em Problemas na Educação a Distância e Híbrida." In Anais do XXXI Simpósio Brasileiro de Informática na Educação, pp. 61-71.

Perry, G. T. (2005). "Proposta de uma metodologia participativa para o desenvolvimento de software educacional". Universidade Federal do Rio Grande do Sul. Escola de Engenharia. Programa de Pós-Graduação em Engenharia de Produção.

Ribeiro, Luis R. Camargo. (2008). “Aprendizagem baseada em problema (ABP): uma experiência no ensino superior". São Carlos: EduFSCar

Santa-Rosa, J. G., Struchiner, M. (2010). "Design Participativo de um Ambiente Virtual de Aprendizagem de Histologia". Revista Brasileira de Pesquisa em Educação em Ciências, Vol. 10.

Silva, C., Vitório, S. and Maia, M. (2012). "Uma abordagem de Engenharia de Requisitos para Softwares de Gestão do Aprendizado: o caso AMADeUs". Simpósio Brasileiro de Informática na Educação - SBIE.

Souto, Mychelline \& Silva, Carla. (2020). "Um catálogo de requisitos pedagógicos para auxiliar o desenvolvimento de Softwares Educacionais". Brazilian Symposium on Computers in Education (Simpósio Brasileiro de Informática na Educação - SBIE), [S.1.], p. 506, out. 2017. ISSN 2316-6533. Disponível em: <https://www.brie.org/pub/index.php/sbie/article/view/7579>. 
Teixeira, Adriano \& Canabarro, \& Brandão, \& Edemilson, Jorge \& Ramos. (2003). "Software Educacional: O difícil começo". RENOTE. 1. 10.22456/1679-1916.13629.

Wang, F., and Hannafin. M. J. (2005). "Design-based research and technologyenhanced learning environments." Educational technology research and development 53 , no. 4 .

Zhao, Y., Llorente, A. M. P., and Gómez, M. C. S. "Digital competence in higher education research: A systematic literature review." Computers \& Education (2021): 104212 . 